\title{
Muscle wasting: an overview of recent developments in basic research
}

\author{
Sandra Palus • Stephan von Haehling • Jochen Springer
}

Received: 6 August 2014 / Accepted: 7 August 2014 /Published online: 28 August 2014

(C) Springer-Verlag Berlin Heidelberg 2014

\begin{abstract}
The syndrome of cachexia, i.e., involuntary weight loss in patients with underlying diseases, sarcopenia, i.e., loss of muscle mass due to aging, and general muscle atrophy from disuse and/or prolonged bed rest have received more attention over the last decades. All lead to a higher morbidity and mortality in patients, and therefore, they represent a major socio-economic burden for the society today. This minireview looks at recent developments in basic research that are relevant to the loss of skeletal muscle. It aims to cover the most significant publication of last 3 years on the causes and effects of muscle wasting, new targets for therapy development, and potential biomarkers for assessing skeletal muscle mass. The targets include the following: (1) E-3 ligases TRIM32, SOCS1, and SOCS3 by involving the elongin BC ubiquitin-ligase, Cbl-b, culling 7, Fbxo40, MG53 (TRIM72), and the mitochondrial Mul1; (2) the kinase MST1; and (3) the G-protein $\mathrm{G} \alpha \mathrm{i}_{2}$. D(3)-creatine has the potential to be used as a novel biomarker that allows to monitor actual change in skeletal muscle mass over time. In conclusion, significant development efforts are being made by academic groups as well as numerous pharmaceutical companies to identify new target and biomarker muscles, as muscle wasting represents a great medical need, but no therapies have been approved in the last decades.
\end{abstract}

\footnotetext{
S. Palus $\cdot$ S. von Haehling $\cdot$ J. Springer

Department of Innovative Clinical Trials, University Medical Centre Göttingen, Göttingen, Germany

J. Springer $(\bowtie)$

Department of Cardiology and Pneumology, University Medical Centre Göttingen, Robert-Koch-Str. 40, 37075 Göttingen, Germany

e-mail: jochen.springer@charite.de

S. von Haehling

Applied Cachexia Research, Department of Cardiology,

Charité Medical School, Campus Virchow-Klinikum,

Augustenburger Platz 1, 13353 Berlin, Germany
}

Keyword Cachexia sarcopenia $\cdot$ Basic research $\cdot$ Muscle wasting

\section{Introduction}

Cachexia sarcopenia and general wasting of the musculature are related to a poor quality of life and increased morbidity/ mortality [1]. They are caused by a large number of chronic diseases and the general process of aging thus affecting millions of patients and elderly [2-4]. The syndrome of cachexia is characterized as complex metabolic abnormalities that lead to the loss of body weight as a consequence of a chronic illness. A consensus statement from 2008 proposed to clinically define cachexia as a non-edematous weight loss exceeding $5 \%$ within the previous $3-12$ months in combination with symptoms characteristic for cachexia (e.g., fatigue or depression), loss of lean body mass, and biochemical abnormalities (e.g., anemia or inflammation) associated with chronic diseases [5]. In adults, a prevalence of 5-15\% has been reported in chronic heart failure (CHF) and chronic obstructive pulmonary disease (COPD), while it may be up to $80 \%$ in advanced cancer [6]. Interestingly, an estimated $30 \%$ of cancer patients die as a result of cachexia rather than the cancer itself [6], although the precise cause of death due to cachexia is still somewhat unclear.

In contrast to the relatively fast atrophy of skeletal muscle associated with cachexia, the syndrome of sarcopenia is characterized by a much slower decline in muscle mass and function that is directly related to the aging process and may ultimately lead to frailty and loss of independent living [7]. There is a loss of $1-2 \%$ of muscle mass per decade of life from the fifth decade onwards, associated with a $1.5 \%$ decline in muscle strength, potentially increasing to $3 \%$ after the age of 60 [7]. From a histological point of view, sarcopenia is characterized by a decrease in the number and the size of the 
muscle fibers. The prevalence of sarcopenia for those over 64 years of age has been shown to be $22.6 \%$ in women and $26.8 \%$ in men, rising to 31.0 and $52.9 \%$, respectively, in those over 80 years of age [8]. It can thus be estimated that over $3 \%$ of the total world population will be affected by sarcopenia by 2015 [8].

However, muscle wasting may also occur independently of chronic diseases and age. Disuse of muscle is a strong inducer of skeletal muscle atrophy and function that is caused by a mechanical unloading of the muscle, e.g., space flight or prolonged bed rest, and involved multiple signaling pathways [9].

The development of preventive and therapeutic strategies against cachexia, sarcopenia, and wasting disorders in general is perceived as an urgent need by health care professionals $[10,11]$. Despite this great medical need, no therapies have been approved for muscle wasting or cachexia in the last decades. Nevertheless, significant efforts to identify new targets are being made by academic groups as well as numerous pharmaceutical companies [12-14].

\section{Current developments in basic cachexia research}

The mass of a muscle is determined by dynamic regulation of its protein balance in the muscle fibers in response to various extracellular stimuli that can be anabolic or catabolic in nature. These signals may also affect the proliferation and maturation of muscle stem cells. Potent anabolic signals in skeletal muscle are insulin, insulin-like growth factor-1 (IGF-1) [15], testosterone [16], and agonists of the $\beta-2$ adrenoreceptor [17]. Levels of IGF-1 are regulated by the ghrelin/growth hormone axis [18]. Upon binding to the receptor, both insulin and IGF-1 activate the PI3K/Akt/mTOR pathway, which induces protein synthesis while inhibiting proteolysis resulting in a hypertrophy of the muscle [19]. Interestingly, hypogonadism and low testosterone in male cancer patients has been reported to be as high as $90 \%$, thus making these men more susceptible to the development of muscle atrophy [20]. Indeed, a class of molecule termed specific androgen receptor modulators (SARMs) have shown to increase muscle mass in the elderly [21]. Triggers for muscle wasting are more numerous and include a general activation of the sympathetic nervous system (SNS) [22], pro-inflammatory cytokines [23, 24], angiotensin-II [25], glucocorticoids [26, 27], and members of the TGF- $\beta$ family $[28,29]$.

\section{News in catabolic signaling}

In healthy individuals, muscle growth is limited by several members of the TGF- $\beta$ family, namely, myostatin, activin A, and TGF- $\beta$ binding to the activin IIB receptor or the TGF- $\beta$ receptor, respectively. Under disease conditions, these proteins prominently contribute to the induction of protein loss in skeletal muscle $[30,31]$. Activation of either receptor induces SMAD2/SMAD3 signaling resulting in inhibition of anabolic Akt-signaling and stimulation of proteolysis $[19,30]$. Cytokines like interleukine-1 (IL-1), IL-6, TNF- $\alpha$, and interferon- $\gamma$ lead to a receptor-mediated activation of NFKB and FOXO in muscle [32]; the latter can also be activated by glucocorticoids [19]. Among others, the glucocorticoid receptor activates REDD1 and KLF15 expression, both inhibiting mTOR activity, KLF15 via BCAT2 gene activation, and it also directly upregulates the expression of MuRF-1 and MAFbx resulting in atrophy [33]. Interestingly, NFKB-inhibition by targeting the IkappaB kinase complex only partially prevents cachexia [34], suggesting some redundancy in catabolic signaling, and NFKB signaling is activated in cachectic, but not in sarcopenic, muscle [35]. Moreover, IL-1 $\alpha$ and TNF- $\alpha$ block differentiation of human myoblasts into myotubes via TGF- $\beta$-activated kinase-1 (TAK-1), which was dependent on the transcriptional induction of activin $\mathrm{A}$ and its subsequent signaling via activin IIB receptor and downstream activation of SMAD2/SMAD3 [36]. The transcription factors NFKB, FOXO, and SMAD2/SMAD3 are considered to be crucial for promoting proteolysis by inducing the transcription of atrogenes including the muscle-specific E-3 ubiquitin ligases MAFbx and MuRF-1 [37]. Induced expression of these two ligases has been used in synonym with increased activity of the ubiquitin-proteasome system (UPS). While MAFbx mainly targets regulatory proteins involved in controlling protein synthesis, e.g., the initiation factor eIF3-f [38], as well as the transcription factor MyoD that is crucial to maintain the differentiated phenotype of adult fast skeletal muscle fibers [39], MuRF-1 has been shown to target the myofibrilles directly [40]. Sarcomeric proteins are degraded in an ordered process in which MuRF1 catalyzes ubiquitylation of thick filament components like myosin heavy chain [40], which becomes accessible after the thin filaments (actin, tropomyosin, troponins) and Z-band (alpha-actinin) components and have been ubiquitylated by the constitutively active E-3 ligase TRIM32, which promotes their degradation by the $26 \mathrm{~S}$ proteasome [41]. Furthermore, the transcription factor Sox6, which plays an essential role in muscle fiber differentiation by blocking slow fiber associated gene expression, is targeted by the E-3 ligase Trip12 [42]. In C2C12 myotubes, inhibition of Trip 12 or the $26 \mathrm{~S}$ proteasome increased Sox 6 protein resulting in a decrease in slow fiber-specific Myh7 expression, while an increased expression in fast fiber-specific Myh4 was detected [42]. Thus, Trip12 may play an important role in the fiber-type switch observed under atrophic conditions [43].

An additional muscle-specific mechanism of atrophy is the intracellular blunting of the anabolic IGF-1 signaling. Upon activation of the IGF-1 receptor IRS-1 is phosphorylated and induces the $\mathrm{PI} 3 \mathrm{~K} / \mathrm{Akt} / \mathrm{mTOR}$ pathway that induces protein 
synthesis [19]. Previous studies have shown that a number of distinct E3 ubiquitin ligases target IRS-1 under different circumstances. SOCS 1 and SOCS 3 target IRS-1 in inflammation-induced insulin resistance possibly by involving the elongin BC ubiquitin-ligase [44]. Under unloading conditions, i.e., disuse, the ubiquitin ligase Cbl-b is induced and terminates IGF-1 signaling [45]. The culling 7 E3 ligase complex containing the Fbw8-substrate-targeting subunit Skp1 and the ROC1 RING finger protein is thought to play a role in cellular senescence by providing a negative feedback loop to mTOR signaling [46]. Mutated cullin 7 has also been liked to pre and postnatal growth retardation [47]. Recently, Fbxo40 has been described as a new IRS-1 targeting E3 ligases that induces rapid degradation of IRS-1 after stimulation of muscle cells with IGF-1 [48], thereby limiting overall muscle growth by de-sensitizing. Interestingly, the expression of Fbxo40 is muscle specific, being only expressed in myocytes and cardiomyocytes with increasing levels upon differentiation of the muscle cells [48]. The most recent addition to the IRS-1 targeting E3 ligases is muscle-specific mitsugumin 53 (MG53; also called TRIM72), which induces ubiquitination together with the E2 enzyme UBE2H and additionally directly targets the insulin receptor, but not the IGF-1 receptor [49]. Overall, the blunting of IGF-1/ IRS-1 signaling not only leads to an inhibition of protein synthesis with concurrent induction of proteolysis, but IRS-1 itself cannot be regenerated after UPS-mediated degradation, thereby leaving the muscle cells unresponsive [29]. Although insulin resistance is noted in many patients with cancer cachexia [50], IGF-1 itself and the IGF-1 receptor are targeted in cancer patients, as they play a role in the growth of several tumors, including pancreatic cancer, which might further impair anabolic signaling in skeletal muscle [51]. However, in non-IGF-1-dependent cancers, IGF-1 supplementation may reduce muscle wasting [52]. Interestingly, some chemotherapeutic drugs state weight loss as side effects, e.g., the mTOR inhibitor temsirolimus, which increased loss of body fat, suggesting a possible end-organ metabolic effect [53].

While the UPS is considered to be the major proteolytic pathway responsible for the breakdown of muscle proteins [54], induction of FOXO1/3 by catabolic stimuli has also been linked to mitochondrial dysfunction and a subsequent loss of mitochondria in skeletal muscle contributing to impaired muscle function [55]. The mitochondrial E3 ligase 1 (Mul1) promotes the fragmentation, depolarization, and clearance of mitochondria through the autophagy-lysosome pathway termed mitophagy. Mull is upregulated through a mechanism involving FOXO1/3 transcription factors under catabolic conditions [56]. However, markers of mitochondrial function, e.g., pyruvate dehydrogenase function, in colon cancer patients suggest that muscle mass and mitochondrial enzyme activity are not invariably linked [57] and that the reduction muscle mitochondrial oxidative capacities may be linked to a decrease in complex IV activity [58]. In denervation models, Mammalian sterile 20-like kinase 1 (MST1) is highly expressed in skeletal muscle and has been suggested to be a key regulator of muscle atrophy, but only affecting the fast fiber type muscle fibers by FOXO3a-mediated induction of MAFbx and LC3; the latter suggesting an activation of autophagy [59].

In addition, another member of the TGF- $\beta$ family, macrophage inhibitory cytokine-1/growth differentiation factor 15 (MIC-1/GDF15), has been shown to be upregulated by 10 100 -fold in some cancers resulting in anorexia by direct actions of the circulating cytokine on feeding centers in the brain [60].

In summary, novel targets identified in the last few years for anti-catabolic intervention strategies include (1) E-3 ligases TRIM32, SOCS1, and SOCS3 by involving the elongin BC ubiquitin-ligase, Cbl-b, culling 7, Fbxo40, MG53 (TRIM72), and the mitochondrial Mul1 and (2) the kinase MST1.

\section{News in anabolic signaling}

It has been well established that GH, IGF-1, and insulin are potent anabolic factors in skeletal muscle, promoting muscle mass gain. GH primarily regulates liver IGF-1 expression with downstream anabolic effects in skeletal muscle. Insulin and $\mathrm{GH}$ are also involved in fat metabolism: GH induces lipolysis and insulin promotes synthesis of fatty acids in the liver and inhibits their degradation in adipose tissue [15]. However, the GH/IGF-1 axis is controlled by various factors, including ghrelin [61], gender, age, diet, exercise, adiposity, and sleep [15]. Ghrelin and its analogues have been shown to inhibit expression of MAFbx and MuRF1 [62]. Independent of the GH/IGF-1/Akt/mTOR axis, other mechanisms inducing muscle hypertrophy have been described. Commonly used $\beta-2$ adrenoreceptor agonists originally developed for asthma, e.g., formoterol and salbutamol, have been known for some time to have anabolic properties on skeletal muscle via a direct $\beta-2$ adrenoreceptor mechanism that has even let to abuse in professional sports [63]. However, in an experimental cachexia model, formoterol treatment normalized the expression of genes involved in the homeostasis and regeneration of muscle by increasing the expression of Pax7 and follistatin, while decreasing expression of the Sirt1, ActIIB receptor, and myostatin [17, 64]. In combination with the appetite stimulant megestrol acetate, formoterol use in patients with advanced cancer cachexia was well tolerated and muscle mass and/or function were improved to a clinically significant extent in a small phase II study [65].

Aside from the $\beta-2$ adrenoreceptor, there are more Gprotein-coupled receptors (GPCRs) that have anabolic effects when activated. The corticotropin-releasing factor receptor 2 (CRFR2) induces skeletal muscle hypertrophy when activated, particularly by urocortin 3 , which improved muscle function and ameliorated atrophy in several rodent models, 
including disuse, denervation, aging, and muscular dystrophy [66]. Binding of Wnt7a to the GPCR Frizzled7 (Frz7) directly activates the Akt/mTOR growth pathway, thereby inducing myofiber hypertrophy in myotubes. The association of the Fzd7 receptor complex with $\mathrm{G} \alpha_{\mathrm{s}}$ and PI3K was required for this mechanism and Wnt7a-Fzd7 activation of this pathway was completely independent of IGF-receptor activation [67]. The lysophosphatidic acid (LPA) receptor has been known to trigger $\mathrm{Ca}^{2+}$ activation in myotubes and may couple to several G-proteins. However, in human skeletal myotubes, the LPA receptor mainly couples to $\mathrm{G} \alpha \mathrm{i}$, as its effects can be blocked with pertussis toxin, an inhibitor of G $\alpha$ i-mediated signaling [68]. The effects of the LPA receptor were mediated by the $\mathrm{G} \alpha \mathrm{i}_{2}$ G-protein. Interestingly, the overexpression of a constitutively active mutant of ${\mathrm{G} \alpha \mathrm{i}_{2}}_{2}$ induced myotube growth by involving NFAT and PKC and inhibited TNF- $\alpha$-induced atrophy by transcriptional downregulation of MuRF1. Moreover, it was also reported to enhance muscle regeneration and cause a switch to oxidative fibers, making $G \alpha i_{2}$ an interesting drug target independent of any GPCRs [68].

\section{Biomarkers}

A major difficulty in developing anti-wasting therapy strategies and novel drugs is the precise assessment of skeletal muscle mass and any changes during the studies. Currently, wasting assessment is limited to imaging-related quantification of muscle mass by either magnetic resonance imaging (MRI), computed tomography (CT), or dual energy X-ray absorptiometry scan (DEXA) and functional tests to quantify muscle function. Unfortunately, they are all cost-intensive and only available at big medical centers. Furthermore, these methods allow only detection of wasting, but not of patients at risk of developing muscle atrophy [69, 70]. Therefore, the identification of reliable biomarkers that can be measured in plasma and can be used in a cost-effective manner that guide diagnosis and therapy in routine clinical practice and clinical trials is an important part of further drug development [70].

Unfortunately, in the long list of biomarkers that is assessing a specific mechanism of muscle atrophy, many turn out to be not specific to muscle and only weakly associated with clinically relevant outcomes [70]. However, a number of plasma assessable, specific biomarkers to muscle mass have been identified recently. It has been proposed that serological peptide biomarkers whose tissue and pathology specificity are derived from posttranslational modification of proteins in tissues of interest and represent the most accurate tools for identifying novel, meaningful biomarkers [71]. For example, during collagen synthesis in soft lean tissue, $\mathrm{N}$-terminal propeptide of type III procollagen (P3NP) is released into circulation, and its levels have been described to be associated with subsequent changes in lean mass during testosterone and
GH treatment of elderly patients [72]. Testosterone exposure may also increase skeletal muscle expression of the collagen type III gene that encodes P3NP, which was found to be elevated dose-dependently in plasma, making P3NP a biomarker candidate for muscle anabolism [73]. Another collagen fragment, the type VI collagen N-terminal globular domain epitope, which is an MMP-generated degradation fragment of collagen 6 , was tested as a biomarker candidate in a small human immobilization-remobilization study [74]. However, while a correlation was found in young men at baseline and at the end of the study, none was found in elderly [74].

Myostatin seems a natural candidate for an atrophy biomarker as it directly mediates catabolic signaling and is found in plasma [28]. However, the data of a recent study in cardiac cachexia could not confirm a role of circulating myostatin as a biomarker for muscle wasting [75]; thus, more/better validation is needed. Moreover, branched chain amino acids have been suggested as markers, as they are elevated in insulin resistance, although in cancer, they may be unreliable due to the tumor metabolism [76]. Serum creatinine under steady state conditions has been suggested to serve as a reliable muscle mass biomarker, if appropriate adjustment for kidney function and dietary meat intake is undertaken [77].

A recent study used a different approach; rather than trying to identify a new protein/peptide in plasma, it utilized a heavy water-labeled tracer - creatinine-(methyl-d(3)). Its levels in urine were measured after giving rats an oral tracer dose of $\mathrm{D}(3)$-creatine to determine creatine pool size and skeletal muscle mass. It had a very high bioavailability $(>99 \%)$ with low $(0.2-1.2 \%)$ urinary spillage and a steady state after 24 $48 \mathrm{~h}$ [78]. Creatine pool size calculated from urinary D(3)creatinine enrichment at $72 \mathrm{~h}$, significantly decreased with dexamethasone-induced skeletal muscle atrophy and correlated with lean body mass $(r=0.9590 ; c<0.0001)$ [78]. This method has also been tested in humans and provided muscle mass estimates that correlated well with MRI estimates for all subjects $(r=0.868, P<0.0001)$ [79]. Unfortunately, detection requires isotope ratio mass spectrometry (IRMS) or liquid chromatography-tandem mass spectrometry (LC-MS/MS) technology [80], making it a very accurate tool to monitor changes in skeletal muscle mass, but with limited availability.

Acknowledgments The authors of this manuscript certify that they comply with the ethical guidelines for authorship and publishing in the Journal of Cachexia, Sarcopenia, and Muscle 2010; 1:7-8 (von Haehling $\mathrm{S}$, Morley JE, Coats AJ, and Anker SD). This paper is also published in parallel in the International Journal of Cardiology.

\section{References}

1. Kalantar-Zadeh K, Rhee C, Sim JJ, Stenvinkel P, Anker SD, Kovesdy CP. Why cachexia kills: examining the causality of poor outcomes in wasting conditions. J Cachexia Sarcopenia Muscle. 2013;4:89-94. 
2. von Haehling S, Morley JE, Anker SD. From muscle wasting to sarcopenia and myopenia: update 2012. J Cachexia Sarcopenia Muscle. 2012;3:213-7.

3. von Haehling S, Anker SD. Cachexia vs obesity: where is the real unmet clinical need? J Cachexia Sarcopenia Muscle. 2013;4:245-6.

4. Farkas J, von Haehling S, Kalantar-Zadeh K, Morley JE, Anker SD, Lainscak M. Cachexia as a major public health problem: frequent, costly, and deadly. J Cachexia Sarcopenia Muscle. 2013;4:173-8.

5. Evans WJ, Morley JE, Argiles J, Bales C, Baracos V, Guttridge D, et al. Cachexia: a new definition. Clin Nutr. 2008;27:793-9.

6. von Haehling S, Anker SD. Cachexia as a major underestimated and unmet medical need: facts and numbers. J Cachexia Sarcopenia Muscle. 2010;1:1-5.

7. von Haehling S, Morley JE, Anker SD. An overview of sarcopenia: facts and numbers on prevalence and clinical impact. J Cachexia Sarcopenia Muscle. 2010;1:129-33.

8. Iannuzzi-Sucich M, Prestwood KM, Kenny AM. Prevalence of sarcopenia and predictors of skeletal muscle mass in healthy, older men and women. J Gerontol A Biol Sci Med Sci. 2002;57:M772-7.

9. Brooks NE, Myburgh KH. Skeletal muscle wasting with disuse atrophy is multi-dimensional: the response and interaction of myonuclei, satellite cells and signaling pathways. Front Physiol. 2014;5:99.

10. Marzetti E, Calvani R, Cesari M, Buford TW, Lorenzi M, Behnke BJ, et al. Mitochondrial dysfunction and sarcopenia of aging: from signaling pathways to clinical trials. Int J Biochem Cell Biol. 2013;45: 2288-301.

11. Haran PH, Rivas DA, Fielding RA. Role and potential mechanisms of anabolic resistance in sarcopenia. J Cachexia Sarcopenia Muscle. 2012;3:157-62.

12. Abstracts of the 7th cachexia conference, Japan, december 9-11, 2013. J Cachexia Sarcopenia Muscle. 2013;4:295-343.

13. Abstracts of the 7th cachexia conference, kobe/osaka, Japan, december 9-11, 2013 (part 2). J Cachexia Sarcopenia Muscle. 2014;5:3578 .

14. Vaughan VC, Martin P, Lewandowski PA. Cancer cachexia: impact, mechanisms and emerging treatments. J Cachexia Sarcopenia Muscle. 2013;4:95-109.

15. Trobec K, von Haehling S, Anker SD, Lainscak M. Growth hormone, insulin-like growth factor 1 , and insulin signaling-a pharmacological target in body wasting and cachexia. J Cachexia Sarcopenia Muscle. 2011;2:191-200.

16. Burney BO, Hayes TG, Smiechowska J, Cardwell G, Papusha V, Bhargava $\mathrm{P}$, et al. Low testosterone levels and increased inflammatory markers in patients with cancer and relationship with cachexia. J Clin Endocrinol Metab. 2012;97:E700-9.

17. Busquets S, Toledo M, Marmonti E, Orpi M, Capdevila E, Betancourt A, et al. Formoterol treatment downregulates the myostatin system in skeletal muscle of cachectic tumour-bearing rats. Oncol Lett. 2012;3:185-9.

18. Akamizu T, Kangawa K. Ghrelin for cachexia. J Cachexia Sarcopenia Muscle. 2010;1:169-76.

19. Schiaffino S, Mammucari C. Regulation of skeletal muscle growth by the IGF1-Akt/PKB pathway: insights from genetic models. Skelet Muscle. 2011;1:4

20. Burney BO, Garcia JM. Hypogonadism in male cancer patients. J Cachexia Sarcopenia Muscle. 2012;3:149-55.

21. Dalton JT, Barnette KG, Bohl CE, Hancock ML, Rodriguez D, Dodson ST, et al. The selective androgen receptor modulator GTx024 (enobosarm) improves lean body mass and physical function in healthy elderly men and postmenopausal women: results of a doubleblind, placebo-controlled phase II trial. J Cachexia Sarcopenia Muscle. 2011;2:153-61.

22. Springer J, Tschirner A, Haghikia A, von Haehling S, Lal H, Grzesiak A, et al. Prevention of liver cancer cachexia-induced cardiac wasting and heart failure. Eur Heart J. 2014;35:932-41.
23. Pedroso FE, Spalding PB, Cheung MC, Yang R, Gutierrez JC, Bonetto A, et al. Inflammation, organomegaly, and muscle wasting despite hyperphagia in a mouse model of burn cachexia. J Cachexia Sarcopenia Muscle. 2012;3:199-211.

24. Puppa MJ, White JP, Velazquez KT, Baltgalvis KA, Sato S, Baynes JW, et al. The effect of exercise on IL-6-induced cachexia in the Apc (Min/+) mouse. J Cachexia Sarcopenia Muscle. 2012;3:117-37.

25. Rezk BM, Yoshida T, Semprun-Prieto L, Higashi Y, Sukhanov S, Delafontaine P. Angiotensin II infusion induces marked diaphragmatic skeletal muscle atrophy. PLoS ONE. 2012;7:e30276.

26. Elkina Y, Palus S, Tschirner A, Hartmann K, von Haehling S, Doehner $\mathrm{W}$, et al. Tandospirone reduces wasting and improves cardiac function in experimental cancer cachexia. Int J Cardiol. 2013;170:160-6.

27. Olde Engberink RH, Knippels MC, Pijpers E. Hypomanic episode as a first presentation of a large B-cell lymphoma. Jpn J Clin Oncol. 2013;43:318-20.

28. Elkina Y, von Haehling S, Anker SD, Springer J. The role of myostatin in muscle wasting: an overview. J Cachexia Sarcopenia Muscle. 2011;2:143-51.

29. Egerman MA, Glass DJ. Signaling pathways controlling skeletal muscle mass. Crit Rev Biochem Mol Biol. 2014;49:59-68.

30. Zhou X, Wang JL, Lu J, Song Y, Kwak KS, Jiao Q, et al. Reversal of cancer cachexia and muscle wasting by ActRIIB antagonism leads to prolonged survival. Cell. 2010;142:531-43.

31. Busquets S, Toledo M, Orpi M, Massa D, Porta M, Capdevila E, et al. Myostatin blockage using actRIIB antagonism in mice bearing the Lewis lung carcinoma results in the improvement of muscle wasting and physical performance. J Cachexia Sarcopenia Muscle. 2012;3: $37-43$.

32. De Larichaudy J, Zufferli A, Serra F, Isidori AM, Naro F, Dessalle K, et al. TNF-alpha- and tumor-induced skeletal muscle atrophy involves sphingolipid metabolism. Skelet Muscle. 2012;2:2.

33. Shimizu N, Yoshikawa N, Ito N, Maruyama T, Suzuki Y, Takeda S, et al. Crosstalk between glucocorticoid receptor and nutritional sensor mTOR in skeletal muscle. Cell Metab. 2011;13:170-82.

34. Der-Torossian H, Wysong A, Shadfar S, Willis MS, McDunn J, Couch ME. Metabolic derangements in the gastrocnemius and the effect of compound A therapy in a murine model of cancer cachexia. J Cachex Sarcopenia Muscle. 2013;4:145-55.

35. Sakuma K, Yamaguchi A. Sarcopenia and cachexia: the adaptations of negative regulators of skeletal muscle mass. J Cachexia Sarcopenia Muscle. 2012;3:77-94.

36. Trendelenburg AU, Meyer A, Jacobi C, Feige JN, Glass DJ. TAK-1/ p38/nNFkappaB signaling inhibits myoblast differentiation by increasing levels of activin A. Skelet Muscle. 2012;2:3.

37. Glass DJ. Signaling pathways perturbing muscle mass. Curr Opin Clin Nutr Metab Care. 2010;13:225-9.

38. Lagirand-Cantaloube J, Offner N, Csibi A, Leibovitch MP, BatonnetPichon S, Tintignac LA, et al. The initiation factor eIF3-f is a major target for atrogin1/MAFbx function in skeletal muscle atrophy. EMBO J. 2008;27:1266-76.

39. Lagirand-Cantaloube J, Cornille K, Csibi A, Batonnet-Pichon S, Leibovitch MP, Leibovitch SA. Inhibition of atrogin-1/MAFbx mediated MyoD proteolysis prevents skeletal muscle atrophy in vivo. PLoS ONE. 2009;4:e4973.

40. Cohen S, Brault JJ, Gygi SP, Glass DJ, Valenzuela DM, Gartner C, et al. During muscle atrophy, thick, but not thin, filament components are degraded by MuRF1-dependent ubiquitylation. J Cell Biol. 2009; 185:1083-95.

41. Cohen S, Zhai B, Gygi SP, Goldberg AL. Ubiquitylation by Trim32 causes coupled loss of desmin, Z-bands, and thin filaments in muscle atrophy. J Cell Biol. 2012;198:575-89.

42. An CI, Ganio E, Hagiwara N. Trip12, a HECT domain E3 ubiquitin ligase, targets Sox6 for proteasomal degradation and affects fiber type-specific gene expression in muscle cells. Skelet Muscle. 2013;3:11. 
43. Roberts BM, Ahn B, Smuder AJ, Al-Rajhi M, Gill LC, Beharry AW, et al. Diaphragm and ventilatory dysfunction during cancer cachexia. FASEB J. 2013;27:2600-10.

44. Rui L, Yuan M, Frantz D, Shoelson S, White MF. SOCS-1 and SOCS-3 block insulin signaling by ubiquitin-mediated degradation of IRS1 and IRS2. J Biol Chem. 2002;277:42394-8.

45. Nakao R, Hirasaka K, Goto J, Ishidoh K, Yamada C, Ohno A, et al. Ubiquitin ligase $\mathrm{Cbl}-\mathrm{b}$ is a negative regulator for insulin-like growth factor 1 signaling during muscle atrophy caused by unloading. Mol Cell Biol. 2009;29:4798-811.

46. Xu X, Sarikas A, Dias-Santagata DC, Dolios G, Lafontant PJ, Tsai SC, et al. The CUL7 E3 ubiquitin ligase targets insulin receptor substrate 1 for ubiquitin-dependent degradation. Mol Cell. 2008;30:403-14.

47. Sarikas A, Xu X, Field LJ, Pan ZQ. The cullin7 E3 ubiquitin ligase: a novel player in growth control. Cell Cycle. 2008;7:3154-61.

48. Shi J, Luo L, Eash J, Ibebunjo C, Glass DJ. The SCF-Fbxo40 complex induces IRS1 ubiquitination in skeletal muscle, limiting IGF1 signaling. Dev Cell. 2011;21:835-47.

49. Song R, Peng W, Zhang Y, Lv F, Wu HK, Guo J, et al. Central role of E3 ubiquitin ligase MG53 in insulin resistance and metabolic disorders. Nature. 2013;494:375-9.

50. Honors MA, Kinzig KP. The role of insulin resistance in the development of muscle wasting during cancer cachexia. J Cachexia Sarcopenia Muscle. 2012;3:5-11.

51. Fogelman DR, Holmes H, Mohammed K, Katz MH, Prado CM, Lieffers J, et al. Does IGFR1 inhibition result in increased muscle mass loss in patients undergoing treatment for pancreatic cancer? J Cachexia Sarcopenia Muscle. 2014. doi:10.1007/s13539-014-0145-y.

52. Schmidt K, von Haehling S, Doehner W, Palus S, Anker SD, Springer J. IGF-1 treatment reduces weight loss and improves outcome in a rat model of cancer cachexia. J Cachexia Sarcopenia Muscle. 2011;2:105-9.

53. Veasey-Rodrigues H, Parsons HA, Janku F, Naing A, Wheler JJ, Tsimberidou AM, et al. A pilot study of temsirolimus and body composition. J Cachexia Sarcopenia Muscle. 2013;4:259-65.

54. Han HQ, Zhou X, Mitch WE, Goldberg AL. Myostatin/activin pathway antagonism: molecular basis and therapeutic potential. Int J Biochem Cell Biol. 2013;45:2333-47.

55. Romanello V, Guadagnin E, Gomes L, Roder I, Sandri C, Petersen Y, et al. Mitochondrial fission and remodelling contributes to muscle atrophy. EMBO J. 2010;29:1774-85.

56. Lokireddy S, Wijesoma IW, Teng S, Bonala S, Gluckman PD, McFarlane $\mathrm{C}$, et al. The ubiquitin ligase Mull induces mitophagy in skeletal muscle in response to muscle-wasting stimuli. Cell Metab. 2012;16:613-24.

57. Phillips BE, Smith K, Liptrot S, Atherton PJ, Varadhan K, Rennie $\mathrm{MJ}$, et al. Effect of colon cancer and surgical resection on skeletal muscle mitochondrial enzyme activity in colon cancer patients: a pilot study. J Cachexia Sarcopenia Muscle. 2013;4:71-7.

58. Julienne CM, Dumas JF, Goupille C, Pinault M, Berri C, Collin A, et al. Cancer cachexia is associated with a decrease in skeletal muscle mitochondrial oxidative capacities without alteration of ATP production efficiency. J Cachexia Sarcopenia Muscle. 2012;3:265-75.

59. Wei B, Dui W, Liu D, Xing Y, Yuan Z, Ji G. MST1, a key player, in enhancing fast skeletal muscle atrophy. BMC Biol. 2013;11:12.

60. Tsai VW, Husaini Y, Manandhar R, Lee-Ng KK, Zhang HP, Harriott $\mathrm{K}$, et al. Anorexia/cachexia of chronic diseases: a role for the TGFbeta family cytokine MIC-1/GDF15. J Cachexia Sarcopenia Muscle. 2012;3:239-43.

61. Palus S, von Haehling S, Doehner W, Datta R, Zhang J, Dong JZ, et al. Effect of application route of the ghrelin analog BIM-28131 (RM-131) on body weight and body composition in a rat heart failure model. Int J Cardiol. 2013;168:2369-74.
62. Lenk K, Palus S, Schur R, Datta R, Dong J, Culler MD, et al. Effect of ghrelin and its analogues, BIM-28131 and BIM-28125, on the expression of myostatin in a rat heart failure model. J Cachexia Sarcopenia Muscle. 2013;4:63-9.

63. Prather ID, Brown DE, North P, Wilson JR. Clenbuterol: a substitute for anabolic steroids? Med Sci Sports Exerc. 1995;27:1118-21.

64. Toledo M, Busquets S, Ametller E, Lopez-Soriano FJ, Argiles JM. Sirtuin 1 in skeletal muscle of cachectic tumour-bearing rats: a role in impaired regeneration? J Cachexia Sarcopenia Muscle. 2011;2:57-62.

65. Greig CA, Johns N, Gray C, MacDonald A, Stephens NA, Skipworth RJ, et al. Phase I/II trial of formoterol fumarate combined with megestrol acetate in cachectic patients with advanced malignancy. Support Care Cancer. 2014;22:1269-75.

66. Berdeaux R, Stewart R. cAMP signaling in skeletal muscle adaptation: hypertrophy, metabolism, and regeneration. Am J Physiol Endocrinol Metab. 2012;303:E1-17.

67. von Maltzahn J, Bentzinger CF, Rudnicki MA. Wnt7a-Fzd7 signalling directly activates the Akt/mTOR anabolic growth pathway in skeletal muscle. Nat Cell Biol. 2012;14:186-91.

68. Minetti GC, Feige JN, Rosenstiel A, Bombard F, Meier V, Werner A, et al. Galphai2 signaling promotes skeletal muscle hypertrophy, myoblast differentiation, and muscle regeneration. Sci Signal. 2011;4:ra80.

69. Scharf G, Heineke J. Finding good biomarkers for sarcopenia. J Cachexia Sarcopenia Muscle. 2012;3:145-8.

70. Cesari M, Fielding RA, Pahor M, Goodpaster B, Hellerstein M, van Kan GA, et al. Biomarkers of sarcopenia in clinical trialsrecommendations from the International Working Group on Sarcopenia. J Cachexia Sarcopenia Muscle. 2012;3:181-90.

71. Nedergaard A, Karsdal MA, Sun S, Henriksen K. Serological muscle loss biomarkers: an overview of current concepts and future possibilities. J Cachexia Sarcopenia Muscle. 2013;4:1-17.

72. Bhasin S, He EJ, Kawakubo M, Schroeder ET, Yarasheski K, Opiteck GJ, et al. N-terminal propeptide of type III procollagen as a biomarker of anabolic response to recombinant human GH and testosterone. J Clin Endocrinol Metab. 2009;94:4224-33.

73. Chen F, Lam R, Shaywitz D, Hendrickson RC, Opiteck GJ, Wishengrad $\mathrm{D}$, et al. Evaluation of early biomarkers of muscle anabolic response to testosterone. J Cachexia Sarcopenia Muscle. 2011;2:45-56.

74. Nedergaard A, Sun S, Karsdal MA, Henriksen K, Kjaer M, Lou Y, et al. Type VI collagen turnover-related peptides-novel serological biomarkers of muscle mass and anabolic response to loading in young men. J Cachexia Sarcopenia Muscle. 2013;4:267-75.

75. Christensen HM, Kistorp C, Schou M, Keller N, Zerahn B, Frystyk J, et al. Prevalence of cachexia in chronic heart failure and characteristics of body composition and metabolic status. Endocrine. 2013;43: 626-34.

76. O'Connell TM. The complex role of branched chain amino acids in diabetes and cancer. Metabolites. 2013;3:931-45.

77. Patel SS, Molnar MZ, Tayek JA, Ix JH, Noori N, Benner D, et al. Serum creatinine as a marker of muscle mass in chronic kidney disease: results of a cross-sectional study and review of literature. J Cachexia Sarcopenia Muscle. 2013;4:19-29.

78. Stimpson SA, Turner SM, Clifton LG, Poole JC, Mohammed HA, Shearer TW, et al. Total-body creatine pool size and skeletal muscle mass determination by creatine-(methyl-D3) dilution in rats. J Appl Physiol (1985). 2012;112:1940-8.

79. Clark RV, Walker AC, O'Connor-Semmes RL, Leonard MS, Miller RR, Stimpson SA, et al. Total body skeletal muscle mass: estimation by creatine (methyl-d3) dilution in humans. J Appl Physiol. 1985;2014:1605-13.

80. Stimpson SA, Leonard MS, Clifton LG, Poole JC, Turner SM, Shearer TW, et al. Longitudinal changes in total body creatine pool size and skeletal muscle mass using the D-creatine dilution method. J Cachex Sarcopenia Muscle. 2013;4:217-23. 